\title{
LeGes
}

Ettore Mjölsnes

\section{Plurilinguismo istituzionale e traduzione automatica: verso lingue ufficiali mute?}

Il presente contributo analizza criticamente i programmi di traduzione automatica, mostrando che i testi da essi creati sono il risultato di una mera commutazione di grafemi, priva di senso intrinseco. In quanto tali, essi sono antitetici rispetto alla traduzione umana, che porta invece sempre in sé il riflesso del contesto da cui scaturisce. Per uno Stato plurilingue come la Svizzera, impostato su una concezione equitativa delle lingue ufficiali, il ricorso acritico a queste tecnologie presenta il pericolo di insinuare una visione superficiale e liquidatoria del plurilinguismo che in ultima analisi approda a lingue ufficiali mute.

Categoria di articoli: Resoconti dell'attività

Citazione: Ettore Mjölsnes, Plurilinguismo istituzionale e traduzione automatica: verso lingue ufficiali mute?, in: LeGes 32 (2021) 2 


\section{Indice}

1. La magìa della macchina

2. La magìa della diversità

3. La superficie e il riflesso del testo

4. La profondità della parola

5. Lo splendore del nulla

6. Solo uno strumento per dirozzare il testo?

7. Revisione e post-editing

8. È traduzione?

9. Plurilinguismo istituzionale: quo vadis?

«Imparare una lingua è come piantare un alberello. (...) La pianta ha attecchito, si è ingrandita, ho potuto leggere e poi tradurre, che è un portare prima fiore, poi frutto».

(Erri De LucA ${ }^{1}$ )

\section{La magìa della macchina}

[1] Uscito ormai trent'anni fa, nel 1991, il film «Terminator 2» di James Cameron narra un mondo distopico in cui un sistema informatico resosi autonomo pianifica lo sterminio della razza umana. Più o meno a metà del film i tre protagonisti, in fuga dall'androide programmato dal software per eliminarli, si fermano in Messico in un campo base segreto dell'organizzazione di resistenza creatasi per lottare contro i piani del software. Il terzetto è composto da Sarah Connor, una donna con trascorsi paramilitari decisa a lottare contro l'esercito delle macchine, il suo figlio undicenne John e un androide «buono» (cui Arnold Schwarzenegger ha dato i celebri connotati fisici), programmato per proteggere madre e figlio dall'androide "cattivo». Inizia qui una lunga sequenza in cui la madre, osservando il figlio giocare con l'androide, riflette con una certa amarezza sul fatto che l'androide sarebbe un padre perfetto, a perfect father: un padre che non si stanca mai di giocare con il ragazzo, di rispondere alle sue domande e di trasmettergli le proprie conoscenze; che non perde mai la pazienza, non bestemmia, non torna a casa ubriaco dopo una serata allegra; e che non abbandona moglie e figlio, come invece ha fatto il padre di John.

[2] Da un po' di tempo a questa parte, da quando cioè le sequenze di grafemi che produce hanno superato il livello claudicante dei primi anni, la traduzione automatica appare avvolta dalla medesima aura. Un traduttore che in pochi secondi traduce centinaia o anche migliaia di pagine da qualsiasi lingua in qualsiasi altra lingua, senza mai stancarsi, in qualsiasi ora del giorno o della notte, senza chiedere aumenti di salario, compensazione del lavoro domenicale o rimborso delle spese professionali, senza litigare con i mandanti sulle scadenze e senza importunare gli autori con domande sulle ambiguità e contraddizioni dei loro testi di partenza: potrebbe essere il sogno di ogni responsabile aziendale di comunicazione, marketing e finanze.

[3] Ma è anche più di questo: è un sogno antichissimo dell'umanità fattosi per così dire carne. Come noto, nell'Antico testamento ${ }^{2}$ si narra che in tempi remoti l'umanità parlasse una sola e identica lingua. A un certo punto della storia, un progresso tecnologico - l'impiego di mattoni al

De Luca 2021, pag. 55-56.

Genesi 11, 1-9. 
posto delle pietre e di bitume invece della calce - permette di costruire edifici molto più grandi di prima, e l'umanità decide di edificare una torre alta fino al cielo, l'Etemenanki o Torre di Babele. Il Signore, sceso a osservare questo cantiere, per stroncare negli uomini la mania di grandezza che determina ormai le loro aspirazioni, decide di «confondere» la loro lingua, in maniera che non possano più capirsi tra loro, e li disperde in tutto il mondo. Non è questa la sede per dare un'interpretazione esaustiva di questo passaggio chiave; importa sottolineare che in questo passaggio si manifesta una visione negativa della pluralità delle lingue come punizione divina intesa a ostacolare la realizzazione delle ambizioni dell'umanità e la comunicazione e cooperazione tra gli esseri umani. Una visione negativa peraltro profondamente radicata nei nostri schemi cognitivi ed emotivi, di cui molti bambini plurilingui ne conoscono una delle manifestazioni tipiche, cioè la situazione in cui un adulto (di solito monolingue o, se plurilingue, in rapporto conflittuale con il proprio plurilinguismo) esprime nei loro confronti la convinzione che chi cresce con più di una lingua per forza di cose finirà con l'avere competenze linguistiche insufficienti in tutte le lingue da lui parlate. È la stessa visione negativa che si manifesta nel detto «traduttore traditore», nella diffusa immagine cioè della traduzione come attività deficitaria, in qualche modo sospetta e comunque imperfetta: un male necessario, insomma, da cui sarebbe buona cosa redimere l'umanità ${ }^{3}$.

[4] Così, a parte gli scopi puramente commerciali perseguiti con questa operazione, la traduzione automatica promette en passant anche di realizzare un sogno per millenni considerato irrealizzabile: permettere all'uomo di superare le barriere linguistiche, moltiplicare la quantità di informazioni disponibili in sempre più lingue diverse in sempre meno tempo, diminuire i costi della traduzione, e in generale abolire tutta la «fatica» del tradurre. Non sembrano esserci limiti. L'entusiasmo è comprensibile e spiega forse come mai l'avvento e la diffusione generalizzata di questa tecnologia vengono già oggi visti come un dato di fatto inarrestabile, a prescindere dalle sue implicazioni, come se un fiume in piena scendesse a valle trascinando con sé tutto quanto incontra sul suo cammino.

[5] In tale euforia generalizzata, rilievi critici sembrano giungere soprattutto dagli addetti ai lavori. Per quanto il loro scetticismo venga talvolta attribuito a preoccupazioni di ordine puramente occupazionale $^{4}$, ciò non toglie che essi a ragione attirano l'attenzione sulle imperfezioni sintattiche e semantiche di cui ancora soffre la tecnologia e invitano a una certa prudenza, nell'attesa che questi difetti siano eliminati.

[6] Non vi è dubbio che potranno esserlo. Se incaricati di perfezionare le loro macchine (e dotati delle necessarie risorse finanziarie), i programmatori sapranno applicare algoritmi ancora più complessi, integrare server ancora più potenti e alimentare la macchina con raccolte ancora più ricche di capolavori delle letterature e della multimedialità mondiali. Un bel giorno il software saprà produrre traduzioni davvero «ineccepibili», esprimendosi se necessario in perfetti endecasillabi, imitando a scelta, mediante selezione con tasto destro del mouse, lo stile di Petrarca, Foscolo o di una pubblicità per pneumatici. Il traduttore automatico avrà allora definitivamente

3 Su questa visione negativa, di cui si può cogliere un riflesso anche nella nozione di «residuo traduttivo", cioè nell'idea che vi è sempre una parte di contenuto del testo originale che la traduzione non riesce a rendere nella lingua d'arrivo, cfr. EgGer 2019, pagg. 66 segg, 302 segg.

4 Così Nussbaumer 2020, che attribuisce l'atteggiamento negativo di parte dei traduttori nei confronti della traduzione automatica alla preoccupazione «Kommen da Maschinen, die mich früher oder später ersetzen werden?». 
superato il cosiddetto test di Turing ${ }^{5}$, e sarà diventato il traduttore perfetto che sognavamo, the perfect translator.

\section{La magìa della diversità}

[7] Se l'episodio della torre di Babele veicola un'immagine negativa della pluralità delle lingue, nella Bibbia vi sono anche brani che invece ne danno un'immagine positiva. L'episodio del miracolo pentecostale ${ }^{6}$, ad esempio, in cui la facoltà di esprimersi in più lingue è vista come un dono, non un castigo, divino; o, più indirettamente, l'intero vangelo di Marco, in cui le frequenti locuzioni originali in aramaico (alcune delle quali, come Eloì Eloì, lemà sabactàni ${ }^{7}$, entrate nell'immaginario collettivo), seguite di volta in volta dalla traduzione, fanno della compresenza di lingue diverse un elemento poetico ed icastico che dà corpo e sostanza al tessuto linguistico del testo.

[8] Il fatto che le Scritture presentino due aspetti diametralmente opposti del plurilinguismo non è una contraddizione. Non si tratta infatti di contrapporre una visione all'altra, ma di abbracciarle entrambe. La traduzione è fatica e ostacolo, è «punizione», ma è anche superamento, elevazione, creatività e pienezza, è dono; i due aspetti coesistono, ed è proprio la loro coesistenza a determinarne la ricchezza espressiva e comunicativa a tutti i livelli, inclusi quello economico, culturale e sociale. In termini aziendali si potrebbe dire che l'onere legato alla traduzione è il prezzo da pagare per ottenerne il plusvalore. In una prospettiva più ampia si potrebbe dire che la traduzione, e il plurilinguismo che ne è la precondizione, nella loro complessità non banale ma contraddittoria sono un riflesso fedele e intimo della condizione umana, che non è quasi mai banale e spesso contraddittoria. Una visione differenziata che trova piena espressione nelle sette tesi sulla traduzione enunciate da JEAN-Luc EGGER, secondo cui la traduzione è aumento di informazione, un atto di civiltà, una prova di vitalità di una lingua, la base dell'esistenza di realtà plurilingue, un'opera creativa e un fattore di umanità, ma anche e non da ultimo un'attività, appunto, complessa ${ }^{8}$.

[9] Abbiamo visto che la tecnologia del traduttore automatico promette di eliminare la complessità della traduzione: c'è da chiedersi allora se insieme ad essa non eliminerà anche tutti gli altri suoi elementi. Altrimenti detto, la domanda da porsi non è se l'informatica riuscirà a dare i natali a una macchina in grado di applicare parametri di calcolo tali da permetterle di tradurre come un essere umano, bensì che cosa significa per l'essere umano, in quanto soggetto parlante una o più lingue e in quanto inserito in una società plurilingue, il fatto di poter disporre di una macchina di questo tipo? Anche ammesso (ma non concesso) che a livello testuale non vi sia nessuna differenza apparente tra la traduzione fatta da un uomo e quella fatta da una macchina, l'atto traduttivo è iscritto nella stessa natura umana e, dunque, ha «implicazioni politiche, culturali, scientifiche ed umane» ${ }^{9}$ che vanno ben oltre il livello testuale. Queste implicazioni sono tanto più fondamentali

5 Con questa espressione si designa comunemente una situazione fittizia in cui una persona A viene incaricata di formulare a libera scelta domande a un interlocutore sconosciuto B - di cui A sa soltanto che potrebbe essere un uomo o una macchina - e di decidere, sulla base delle risposte fornite da $B$, se questi è un essere umano o una macchina; il test si intende "superato" dalla macchina che non sia riconosciuta come tale da A: essa potrà allora essere considerata, secondo la terminologia degli ideatori del test, «intelligente».

6 Atti 2, 1-11.

7 «Dio mio, Dio mio, perché mi hai abbandonato?», Marco 15, 34.

8 Egger 2019, pagg. 66-78.

9 EgGer 2019, pag. 66. 
nel caso specifico della Svizzera in quanto realtà sociale e istituzionale plurilingue, e non da ultimo nel caso dell'italiano in Svizzera, che nella triade delle lingue ufficiali federali è quella più fortemente caratterizzata dalla componente traduttiva ${ }^{10}$.

[10] L'evoluzione tecnologica non è un evento ineludibile su cui l'uomo non può influire, ma è il risultato di una scelta, e non può esimersi dall'esserlo, visto che è per forza di cose il risultato di una volontà costruttiva ${ }^{11}$. Ed è compito della società civile valutare con occhio critico ogni innovazione tecnologica prima di permetterne l'uso generalizzato. Nei casi in cui questa valutazione non è stata fatta, le conseguenze sono state a volte nefaste e, soprattutto, sono spesso andate a carico non della generazione che ha introdotto la tecnologia ma di quelle successive.

[11] Si tratta quindi di dare una risposta riflessiva a un quesito tecnologico. Per ovvi motivi, chi vuole promuovere commercialmente i software di traduzione non ha nessun interesse a che si discuta di questo aspetto, ma questo, e non la capacità o no del software di superare il test di Turing, è l'unico aspetto significativo per il nostro giudizio su di esso. Come mostra la storia di un Paese come la Svizzera, che da sempre ha dovuto cercare soluzioni a livello istituzionale per affrontare la realtà multilingue al suo interno ${ }^{12}$, la risposta politica a tale quesito non è assoluta $\mathrm{e}$ invariabile ma va rinegoziata in continuazione ${ }^{13}$. A tal fine è però necessario prendere coscienza delle vere implicazioni del dibattito. Il presente testo si prefigge di contribuire a questa presa di coscienza formulando alcuni termini di fondo della riflessione.

\section{La superficie e il riflesso del testo}

[12] Torniamo però al manufatto concreto in questione: la traduzione. Abbiamo detto che nel dibattito le voci critiche sinora si sono concentrate sull'analisi della superficie della stessa. Al di là dei singoli errori sintattici o semantici in cui incorre il software, a livello della superficie testuale appare anche una differenza molto più fondamentale tra traduzione umana e traduzione automatica.

[13] Se opera di un essere umano, la traduzione è infatti soltanto il risultato visibile di un lungo percorso che si compone di elementi in parte specifici al singolo traduttore e in parte condivisi con tutti i parlanti la lingua di partenza e la lingua di arrivo. Concretamente, in ambito istituzionale la traduzione comporta una serie di interazioni ad almeno tre livelli: innanzitutto vi è il bagaglio culturale, identitario e linguistico personale del traduttore, il percorso biografico e professionale che l'ha portato a redigere la traduzione in questione, le sue competenze e motivazioni - e magari, dopo anni di attività professionale, pure il suo scoramento e i suoi dubbi - e i suoi limiti; in secondo luogo il complesso sistema di strutture istituzionali e processi organizzativi in cui il traduttore è inserito; e infine il contesto storico specifico e le ricadute economiche, culturali e

10 Sul rapporto tra lo statuto dell'italiano come lingua legislativa originale della Confederazione e i processi traduttivi che portano alla effettiva redazione di leggi in tale lingua cfr. EgGer 2019, pagg. 34 segg. (con rimandi in particolare a SNOzzi 2005). Al riguardo si vedano anche ad es. EgGer/Grandi 2013, pag. 215 e Pini 2017 , pag. 94. Sul punto cfr. Balzaretti 2021, pag. 8: «L'invenzione presuppone che si trascenda la logica finita di semplici relazioni determinate tra elementi discreti in presenza, eccedendo l'attuale verso il possibile di un errore che non è semplice negatività logica ma elemento di un nuovo codice in divenire $(. .$.$) ».$

12 Si consideri ad esempio l'evoluzione del ruolo della lingua italiana a livello federale nella Svizzera a partire dal 1848, cfr. Pini 2017 ed Egger 2019, pag. 21 segg.

13 Il carattere continuativo di questa dinamica viene sottolineato da EGGER 2019, pag. 301, secondo cui «[q]uello del plurilinguismo istituzionale è infatti un cantiere permanente (...)». 
sociali legate all'attività dell'istituzione. Tutto ciò concorre a creare il contesto in cui opera il traduttore ${ }^{14}$

[14] Potremmo definirlo il retroterra ${ }^{15}$ della traduzione. Non si intende qui ovviamente una realtà meno importante rispetto al "centro». Il centro - cioè il prodotto finale (la traduzione) (la superficie) - ha il suo peso, è vero, in quanto è il luogo verso cui confluiscono le risorse del retroterra del traduttore, e in quanto tale ha una certa funzione pratica nel legittimare a posteriori l'intero processo traduttivo. Altrimenti detto: le risorse investite hanno portato alla traduzione desiderata. Ma il centro, come è facile capire, non costituisce la ragion d'essere del retroterra da cui trae origine. Quest'ultimo esiste a priori, non è dato alla persona affinché possa tradurre ma, al contrario, la persona traduce poiché e nella misura in cui il suo retroterra glielo consente. Il retroterra è la ragion d'essere della traduzione: e la traduzione riflette il retroterra da cui scaturisce.

[15] La nostra attenzione va quindi posta su questo riflesso $^{16}$, cioè non sul testo che scaturisce dalla traduzione ma sull'intero percorso che porta al singolo testo. In quest'ottica è significativo che nel test di Turing la persona A chiamata a distinguere la macchina dall'essere umano non solo non sappia nulla del suo interlocutore ma nemmeno del contesto di quest'ultimo. L'intera attenzione è posta sulla superficie, sul testo in quanto risultato dell'interazione tra A e B. Il retroterra è completamente assente dal setting del test. E questa assenza non è una svista o una scelta consapevole dovuta alla necessità di semplificare le variabili incluse nel test: è al contrario la condizione necessaria affinché la macchina possa superare il test ${ }^{17}$. Il test «funziona» soltanto in virtù di questo artificio: la macchina riesce a superarlo soltanto se e nella misura in cui l'attenzione si concentra sugli enunciati da essa prodotti, per i quali l'unica condizione è che riescano a somigliare agli enunciati prodotti da un uomo. Il retroterra non conta, conta soltanto che i calcoli applicati dalla macchina producano un'imitazione sufficientemente verosimile.

[16] Ma persino in questa imitazione la macchina si distingue dall'imitazione degli uomini: è un'imitazione monca, l'imitazione di un'imitazione. In un vecchio film di Federico Fellini, Il bidone, Augusto, un uomo di mezza età (interpretato da Broderick Crawford) si spaccia per prete al fine di abbindolare con false promesse gli abitanti creduloni dei paesini che attraversa in auto. Per quanto incallito da una vita spregiudicata, fatta di espedienti e inganni ${ }^{18}$, il falso prete non riesce a sottrarsi a un rimorso e un'inquietudine del tutto umani quando, verso la fine del film, visitando una famiglia di contadini destinata a essere vittima dell'ennesima frode, si trova confrontato con una bambina paralitica che, convinta della bontà e integrità dell'uomo che le sta di fronte, gli chiede di darle la benedizione; questo atto di fiducia, che gli richiama l'immagine

14 È non solo ma anche a questa realtà complessa che si riallacciano le cinque categorie identificate da Franco Buffoni come elementi centrali di ogni processo traduttivo: avantesto, movimento del linguaggio nel tempo, poetica, ritmo e intertestualità (Buffoni 2016, pagg. 15 segg.).

15 La nozione di retroterra intesa in questo senso ci risulta nuova; si riallaccia tuttavia a nozioni esistenti, ad es. a quelle di «splendore» e «fondo» in Max PicArd, cfr. ad es.: «uno splendore circonda la verità» o «il fondo ineffabile delle cose unisce l'uomo con lo stato originario antecedente la parola (...)» (PICARD 2007, pagg. 35 e 74).

16 Sulla nozione di riflesso (Spiegelung) intesa come complementarietà e dipendenza reciproca di elementi solo apparentemente contrapposti, come vita e morte, fede e scienza, amore e odio, ragione e sentimento, uomo e donna, ecc., in virtù delle quali la vita si colma di significato, cfr. Altrichter 2016.

17 Questa lacuna di fondo del test di Turing ne determina un'altra: l'interlocutore A, chiamato a decidere della natura dell'interlocutore $\mathrm{B}$, potrebbe anche non riconoscere la macchina (o riconoscerla) basandosi su supposizioni errate in merito al rapporto esistente tra le risposte che gli vengono fornite e il loro autore: in tal caso, la macchina avrebbe sì superato (o non superato) il test, ma per motivi che non hanno nessun legame reale con il suo output.

18 Come precisa Ugo Balzaretri, «Vorsatz und Irrtum - wie eigentlich das Lügen-Können - sind nicht von ungefähr spezifische Merkmale des Denkens (...)»; in Balzaretri 2018, pag. 666 (corsivo nell'originale). 
della propria figlia con cui ha da poco ristabilito un fragile contatto, induce Augusto a restituire il maltolto alla famiglia della bimba. Augusto è «soltanto» un imitatore, ma lo è a pieno titolo, non soltanto in «superficie»: dietro le sembianze del parroco (falso) si cela un essere umano (vero). L'imitatore è ciò che appare essere per chi è a conoscenza dell'imitazione (gli spettatori del film), cioè appunto un uomo che ha deciso di essere un imitatore ${ }^{19}$. Il riflesso è reale, non è l'imitazione di un riflesso.

[17] Questo riflesso, che è in sostanza la volontà di essere, è la condizione e l'origine di qualsiasi attività umana dotata di senso, anche della lingua in quanto fenomeno originario ${ }^{20}$, e pertanto anche della traduzione. E tale riflesso è totalmente assente dalla traduzione automatica. Se questa constatazione può apparire banale, a maggior ragione stupisce il fatto che essa è quasi totalmente assente dal dibattito sulla traduzione automatica.

\section{La profondità della parola}

[18] Non appena si concentra l'attenzione dai risultati visibili nel testo al retroterra della traduzione, a tutto quanto la precede, la circonda e la segue, estendendo la prospettiva dal mero livello tecnico-materiale al livello del senso che chi parla o scrive vuole conferire agli enunciati linguistici, emerge la differenza fondamentale tra traduzione e traduzione automatica. Per descriverla giova prendere le mosse da una pagina di grande forza espressiva di CAmillo Sbarbaro:

«Capii allora che come a scrivere non m'ero rimesso di proposito, era illusione credere che di proposito potessi smettere; nato da sé, senza mio intervento altro che quello di decifrare e trascrivere, anche questa volta il libretto da sé si sarebbe fatto a mio scapito o vantaggio, non importa se era necessario. Ancora una volta obbedivo: al bisogno di dare espressione a sentimenti e pensieri portati in me da sempre; di alleggerire col mezzo che ho, le parole, debiti di riconoscenza verso persone care, non lasciando per quanto in me che con me ne perisse del tutto il ricordo; di rendere pubbliche grazie a aspetti di luoghi e stagioni che mi furono di conforto, possedendoli meglio col patirli per darne un'immagine; di compiere insomma il ritratto, spero non solo mio, al quale chiamato mi misi da quando fui in grado di esprimermi. Rimasto nelle cose che dico fedele a me stesso, se poi anche nel modo di dirle è vero che mi sono per naturale decantazione spogliato fin dove possibile di letteratura, riaccostandomi alla povertà di 〈Pianissimo〉, avrei assolto il mio compito». ${ }^{21}$

[19] Si potrà obiettare che qui un poeta parla della propria scrittura poetica, mentre i software di traduzione automatica si iscrivono in una logica non letteraria ma organizzativa, amministrativa

19 Il discorso non cambia anche se estendiamo la prospettiva a considerare il fatto che nel film Broderick Crawford in un certo senso «imita» un imitatore, visto che nella vita che conduceva al di fuori di questa pellicola egli non usava visitare paesini spacciandosi per prete. La dinamica si ripete semplicemente a un livello superiore: l'attore che interpreta il falso prete nel film $\grave{e}$ un attore che interpreta un falso prete in un film, non è qualcosa di diverso da sé stesso.

20 Nel senso attribuito da Picard a questo termine, ossia di fenomeno che più di altri dona e manifesta sostanzialità nel mondo.

21 Sbarbaro 1985, pagg. 421-422 (corsivo nostro). 
ed economica, sono un mero ausilio tecnico (torneremo più avanti su questo aspetto) per rendere più efficienti i processi di traduzione e comunicazione.

[20] Ma come è noto la traduzione è scrittura. E anche se la comunicazione aziendale o istituzionale è un processo comunicativo che comprende elementi gestionali e tecnici certamente diversi da quelli che influiscono sui processi letterari, la scrittura in sé, che ne rimane pur sempre l'espressione finale, non è un siffatto elemento. Ogni enunciato linguistico, anche una traduzione, sottostà alla dinamica descritta da SBARBARo, perlomeno se pretende dotarsi di significato, indipendentemente dalla presunta banalità o complessità del suo tema e dalla sua finalità letteraria, pubblicitaria, giuridica o tecnico-amministrativa. «Lo spirito umano non percepisce soltanto l'oggetto come gli si presenta dinanzi; nel suo movimento lo oltrepassa (...) $»^{22}$. Così, anche le parole veicolano in ogni caso molto più del semplice loro significato lessicale: esse sono sempre anche il segno di qualcosa d'altro, che è profondamente radicato nell'essere umano e allo stesso tempo lo trascende. Altrimenti diventano semplice «brusio verbale» ${ }^{23}$.

[21] Chiunque scrive - sia esso uno scrittore, un'istituzione statale che legifera o un'azienda che pubblicizza i propri prodotti - rimane per forza «nelle cose che dice fedele a sé stesso»: nella peggiore delle ipotesi, fedele alla banalità del proprio pensiero e alle lacune delle proprie competenze linguistiche; nella migliore, alla ricchezza e chiarezza delle proprie idee e alla creatività del proprio estro linguistico. Così anche il traduttore: poiché come per sua natura lui medesimo da sé stesso non può prescindere, così non ne può prescindere la traduzione da lui fatta, né quando è fatta bene, né quando è fatta male.

[22] La macchina ovviamente non può essere «fedele a sé stessa», visto che non ha nessun «sé» cui riferirsi, e tanto meno può esserlo la traduzione da essa prodotta. "Automaten stellen nicht nur nichts dar, sie stellen vor allem nicht sich selbst dar» ${ }^{24}$. Il testo tradotto dalla macchina è una semplice serie di grafemi, per quanto selezionati e combinati dalla macchina «intelligentemente», cioè in modo da permettere a un lettore umano di attribuire loro un significato; e anche in questo, l'intelligenza in realtà non sta nella macchina ma nel lettore, che riesce ad attribuire un senso persino a una pura sequenza di segni che non è il frutto di una volontà espressiva ma di un calcolo. Allo stesso modo è soltanto Sarah Connor che può attribuire all'androide le caratteristiche del buon padre, l'androide invece non sta vivendo nessuna esperienza di paternità: esegue semplicemente ordini in base a parametri preprogrammati nei suoi circuiti o, altrimenti detto, «fa» ciò che non può non «fare», anzi ${ }^{25}$, esegue movimenti ciechi - in quanto privi di intenzionalità conformemente al modello operatorio impartitogli.

\section{Lo splendore del nulla}

[23] I testi generati dalla traduzione automatica sono un'illusione ottica, come i trompe-l'oeil che troviamo sulle facciate di molti edifici storici. Anche nel trompe-l'oeil è l'osservatore che attribuisce un senso a una composizione grafica: passiamo sulla strada di fronte a un vecchio palazzo e ci sembra che da una finestra una persona si affacci a guardarci; ma più ci avviciniamo,

22 Picard 2007, pag. 69.

23 Ossia vuoto involucro verbale, secondo la lezione di Max Picard, cfr. Picard 2007, pagg. 153 segg.

24 Altrichter 2020, pag. 63.

25 Giacché non si potrebbe ascrivere all'automa né una praxis né tanto meno una poiesis. 
più ci rendiamo conto che dietro la finestra non sta nessuno e che, anzi, non vi è nemmeno la finestra. Così, pure dietro la pagina tradotta dal software non sta nessuno, e questa assenza è tanto più palese quanto più ci avviciniamo alla finta traduzione.

[24] Ovviamente i trompe-l'oeil non sono soltanto un'illusione ottica, ma anche la manifestazione delle tradizioni delle rispettive maestranze locali, con tecniche, usi e linguaggi pittorici specifici. Anche il software di traduzione automatica potrebbe essere inteso come manifestazione di una «maestranza», quella informatica, e in questa prospettiva testimonierebbe se non altro almeno dell'estro creativo dei suoi programmatori. Ma con una differenza fondamentale: il trompe-l'oeil ha sempre avuto unicamente una funzione estetica, decorativa, e non ha mai voluto sostituirsi al manufatto reale. Il padrone di casa che volesse disporre di una finestra «in carne e ossa» ovviamente non chiederebbe al pittore decoratore di dipingergliene una sulla facciata. Sostituirsi alla traduzione reale è invece proprio quanto pretende di fare la traduzione automatica, o, per essere più precisi, i suoi venditori. Piuttosto sovviene qui un'altra funzione del trompe-l'oeil, che Bruno Donati, parlando della sua diffusione nell'architettura delle valli alpine ticinesi, così descrive: «Un largo impiego di pennelli e colori era riservato ancora una volta agli edifici pubblici più importanti e a chi desiderava mettere in evidenza il proprio ruolo sociale» ${ }^{26}$. Di fronte alla retorica del progresso con cui le presunte potenzialità della traduzione automatica vengono talvolta sbandierate, non ci si può esimere dal pensare che questa euforia rumorosa sia il frutto del medesimo desiderio di evidenziare il proprio statuto sociale che in passato spingeva alcuni padroni di casa a decorare intere facciate con false terrazze, balaustrate, finestre, colonne, nonché allori e stemmi, per impressionare gli ignari passanti.

[25] Ma, qualsiasi siano state le motivazioni del padrone di casa, il trompe-l'oeil, in quanto espressione della bravura del decoratore che lo ha eseguito, è sempre anche fonte di diletto estetico in chi lo osserva, mentre la sensazione suscitata dalla lettura di una traduzione generata da un software è più simile allo sgomento ${ }^{27}$ che si prova vagando in quei non luoghi che sono gli shopping mall di un aeroporto internazionale, avviluppati dall'aria condizionata e dalla luce neon di quell'ambiente lucido, senza spigoli, senza rotture, avvincente, sintetico, freddo, sterile, il cui unico legame con la realtà della vita umana è il fatto di farvi apparizione, apparentemente dal nulla, e di essere dotato di collegamento Internet per le carte di credito (così come il software di traduzione necessita di Internet per produrre i suoi segni).

[26] Come i negozi nelle zone franche doganali degli aeroporti, anche la traduzione automatica si presenta come pienezza allo stato puro; a differenza dei tax-free però, che possono pur sempre servire al viaggiatore annoiato durante il tempo di attesa tra un volo e l'altro a rifornirsi di bibite alcoliche a basso prezzo, l'unica funzione di questa pienezza è quella di coprire, paradossalmente, il vuoto da cui scaturisce, come una foglia di fico che tenta di coprire sé stessa: un tutto colmo di niente. «Das Paradox des Replikanten rührt daher, dass für ihn alles von Bedeutung ist (...), und wiederum nichts, weil er letztlich bloss seine Konstitution reproduziert.» ${ }^{28}$

[27] In quest'ottica appare significativo che alcuni dei software di traduzione automatica disponibili oggi sul mercato discendono da programmi sviluppati durante la guerra fredda dall'esercito americano per permettere alle proprie unità di disporre di traduzioni rapide dei messaggi del-

26 Decorazioni Pittoriche 1998, pag. 7 (corsivo nostro).

27 E, lo sottolineiamo ancora una volta, invece di diminuire, tale sgomento non fa che aumentare più è "perfetta» la superficie della «traduzione» fornita dal software.

28 Altrichter 2016, pag. 82 (corsivo nostro). 
l'esercito sovietico che esse riuscivano a intercettare. Non è una novità che le tecnologie militari fungano da testa di ponte per l'uso civile di applicazioni analoghe, ma ciò che conta sottolineare qui è che questa tecnologia nasce da un contesto di ostilità e diffidenza verso le lingue altre, viste come un ostacolo, uno strumento di segretezza e occultamento il cui codice va decifrato contro la volontà dei suoi utenti originari. Emblematicamente, questa tecnologia, di cui oggi si vantano i presunti vantaggi per la comunicazione linguistica, nasce da un sostrato militare caratterizzato dalla totale assenza della stessa: per poter capire i messaggi dell'Armata rossa infatti, più che incaricare reparti di informatici di sviluppare un software di traduzione, sarebbe stato sufficiente insegnare la lingua russa ai soldati americani. Ma per farlo ci sarebbe voluta un po' più di fiducia nel potenziale plurilingue dell'uomo.

\section{Solo uno strumento per dirozzare il testo?}

[28] A questo punto possiamo esaminare una prerogativa di fondo associata sin dall'inizio all'uso pratico dei software di traduzione, con cui si tenta di riportare la discussione a un livello tecnico-pragmatico: d'accordo, ci viene detto, la macchina non può produrre enunciati dotati di senso, non può creare comunicazione, non può sostituire l'uomo, ma nessuno pretende tanto: la macchina è soltanto un ausilio. Essa funge da mero strumento di lavoro che produce una prima, approssimativa «stesura» della traduzione ${ }^{29}$ : spetta poi agli specialisti della traduzione controllare e, ove necessario, affinare il «prodotto» linguistico finale.

[29] Lungi dal volersi sostituire al traduttore, la macchina si limiterebbe quindi a semplificare il lavoro, evitandogli di «perdere tempo» con la traduzione «vera e propria» e consentendogli di dedicare tutte le sue energie alla revisione delle traduzioni o, nel linguaggio corrente, al post-editing. E tale attività non si distinguerebbe dalla revisione di traduzioni fatte da un collega umano se non per il fatto che il traduttore automatico fornisce la «prima stesura» anche di centinaia di pagine con pochi click nel corso di pochi secondi.

[30] L'avviso figurante nella pagina Internet in cui l'Unione europea mette a disposizione del pubblico il suo software di traduzione automatica è emblematico in proposito: «use it the software] to grasp the gist of a text or as the starting point for a human-quality translation ${ }^{30}$. Un apparato istituzionale autorevole come l'Unione europea ritiene quindi che la traduzione automatica, sebbene non offra alcuna garanzia di correttezza testuale ${ }^{31}$, sia sufficiente per permettere al lettore di afferrare l'essenza (the gist) di un testo oppure (l'opposizione è degna di nota) per fungere da base per una traduzione di qualità umana. Si noti l'aggettivo «umano» aggiunto alla qualifica «di qualità», quasi a dire che anche la traduzione automatica è una traduzione di qualità, semplicemente di una qualità un po' diversa da quella umana. Non a caso l'evidenziazione di questa «umanità» tradisce un'inquietudine, come se il redattore di quelle righe ${ }^{32}$, mosso da un

29 Nell'Amministrazione federale ad esempio l'uso di programmi di traduzione automatici è ammesso, «con una buona dose di cautela», per la traduzione di testi interni non confidenziali per «comprendere un testo nella propria lingua materna o per un uso personale» (promemoria della Cancelleria federale del 19 dicembre 2019 per l'utilizzo di DeepL o di altri programmi di traduzione online).

30 https://ec.europa.eu/info/resources-partners/machine-translation-public-administrations-etranslation_it.

31 Poiché, giova ripeterlo, il software - anche quello che avesse superato il test di Turing - non «sa» che cosa scrive.

32 Ammesso che quelle righe siano state scritte da un essere umano e non dalla macchina. E nel suscitare questo dubbio la pagina ben illustra lo sgomento da aeroporto descritto sopra, che ci induce a chiederci di quali parti del testo ci si possa ancora fidare in quanto autentici. 
sentimento forse non dissimile dal rimorso del falso prete Augusto, avesse sentito la necessità di apporre un piccolo correttivo al miracolo che presenta ai nostri occhi, un'ammissione implicita del fatto che questo colosso ha i piedi d'argilla.

[31] È qui che entra in azione il post-editor, il «fattore umano» cui spetta il compito delicato di aggiungere un po' di malta ai piedi d'argilla affinché il colosso non crolli su chi l'ha costruito: «Das Übersetzen verschiebt sich (...) immer mehr zu einem Post-Editing, zu einem (...) Veredeln dessen, was die Maschine geleistet hat $\rangle^{33}$. A una generazione come la nostra, figlia di varie rivoluzioni industriali, del fordismo e dell'era digitale, questa idea sembra subito familiare: è l'idea della catena di montaggio.

[32] Forse gli ideatori del "post-editing» sono stati indotti in errore dall'affinità del termine italiano «revisione» con il mondo dei motori. Fatto sta che, così come il montaggio di un'automobile in una fabbrica avviene a partire dai suoi singoli pezzi, in una catena il più possibile automatizzata, con controllo di qualità finale da parte di un essere umano, nella figura del post-editor prende forma una modalità produttiva industriale in cui il software assembla i pezzi di base (the gist) della traduzione, su cui interviene poi l'essere umano incaricato del «controllo di qualità». Egli sostituisce, sempre che gli sia concesso il tempo di farlo ${ }^{34}$, un'espressione, sposta magari un aggettivo, un avverbio o una locuzione verbale, riscrive per intero quelle frasi eleganti e perfette in cui immancabilmente il software ha preso fischi per fiaschi: insomma, affina il prodotto del montaggio prima che sia consegnato al cliente, chiavi in mano.

\section{Revisione e post-editing}

[33] Questo modo di vedere le cose misconosce clamorosamente la vera natura della revisione di traduzioni ${ }^{35}$. Tutto il discorso fatto sopra per il traduttore vale tale e quale anche per il revisore: anche questi agisce partendo dal suo retroterra specifico, anche egli è nelle cose che dice sempre fedele a sé stesso, e nella revisione questa sua fedeltà si sovrappone e interagisce con quella del traduttore. Così come c'è una costante interazione tra testo di partenza e prima stesura del traduttore, e poi tra questa e la versione che egli trasmette al proprio revisore ${ }^{36}$, così anche la revisione è per definizione un'operazione ermeneutica, dialogica. Quando i termini di consegna e le modalità di cooperazione lo consentono, il dialogo avviene concretamente tra revisore e traduttore (il revisore consegna le proprie proposte di correzione al traduttore affinché questi possa prendere posizione in merito e dare la forma finale al testo), ma a prescindere da questo aspetto

33 Nussbaumer 2020 (corsivo nostro).

34 A ragione EGGER 2019, pagg. 77-78, menziona le nefaste ripercussioni sulla traduzione della generale accelerazione di ogni cosa.

35 Tralasciamo qui la mera ottica aziendale, secondo cui la revisione delle traduzioni deve in ogni caso seguire un imperativo di economia del lavoro e quindi essere non solo efficace ma anche efficiente. Visto che gli enunciati creati dal software sono privi di senso (come abbiamo visto è il lettore che lo proietta su di essi), la loro revisione richiederebbe l'esame minuzioso di ogni lessema e sintagma per assicurarsi che non vi siano crepe in quella superficie perfetta. Ma di norma il revisore si limiterà a un esame superficiale, nella speranza di individuare gli strafalcioni più appariscenti: l'analisi e la riformulazione di dettaglio richiederebbero infatti un dispendio di tempo tale da equivalere di fatto a nuova traduzione del testo di partenza.

36 Non bisogna dimenticare infatti che a differenza del traduttore automatico, che produce soltanto relazioni determinate e statiche, da lui non modificabili a posteriori, il traduttore in carne e ossa fornisce sempre una traduzione già da lui medesimo rivista, sia che separi le due operazioni in due fasi distinte, sia che proceda rivedendo le proprie frasi man mano che avanza nella traduzione del testo: la traduzione fatta da un essere umano non è pertanto mai una "prima stesura» ma sempre già il risultato di una selezione. 
procedurale esso è già insito nella natura stessa dell'intervento che il revisore fa sul testo: è un dialogo tra la sua forma mentis e quella del collega che ha tradotto, anche quando questi non gli sia noto o non vi sia contatto personale tra i due. Chiunque abbia già fatto revisione di traduzioni sa ad esempio che è possibile identificare lo stile dei singoli colleghi traduttori, o riconoscere se la traduzione è stata fatta da una persona con molta esperienza o da un traduttore alle prime armi $\mathrm{o}$, in un testo tradotto da più persone, identificare il punto esatto in cui è avvenuto il passaggio da un traduttore all'altro, e via dicendo.

[34] La riflessione sullo stile e sulle scelte linguistiche del traduttore non è soltanto un segno di rispetto del revisore verso il collega - cioè un elemento che si ricollega alla dimensione etica insita nella traduzione, sopra evocata, che è sempre anche una lezione di dialogo, rispetto e attenzione reciproci - ma deriva intrinsecamente dalla natura dialettica ed ermeneutica di questo processo. Il software non può spiegare al revisore i motivi delle sue «scelte» terminologiche, richiamandosi ad esempio alla linea redazionale del gruppo di persone con cui lavora, al registro linguistico utilizzato nei testi di riferimento, alla realtà metatestuale (autori e destinatari del testo, funzionalità comunicative dello stesso ecc.), alla tradizione linguistica o a nozioni acquisite nei corsi di formazione seguiti o in un libro letto di recente: paradossalmente, il software, autore prolifico di paginate intere di traduzioni, non ha nulla da dire al suo revisore.

[35] Al confronto risulta essere molto più dialettica la revisione di un motore, in cui vi è effettivamente un'interazione tra uomo e macchina: il motore porta in sé le tracce dell'uso che ne è stato fatto e della manutenzione (o mancante manutenzione) subita, tracce che il meccanico riesce a decifrare e che possono aiutarlo nel formulare la diagnosi del guasto; quando poi il meccanico avrà terminato il suo intervento, pulendo e lubrificando il cuscinetto difettoso, sostituendo la guarnizione rotta o correggendo la posizione delle valvole, il motore revisionato tornerà a funzionare come prima, in alcuni casi anche meglio di prima. La macchina esce cioè trasformata dal contatto con l'uomo, la revisione «le ha dato nuova vita». Non così la traduzione automatica: come il testo prodotto dal software è una traduzione a senso unico, così anche la revisione di un siffatto testo è una revisione a senso unico. La macchina non interagisce in nessun modo con il suo revisore umano, né questi con essa. Anche se la traduzione riveduta confluisce nel corpus su cui la macchina si basa per «imparare», la macchina non riuscirà mai ad acquisire insegnamenti diretti da una singola revisione concreta, anzi, può anche darsi che non ne acquisisca nessuno se in base agli algoritmi applicati tale singola revisione è scartata come base per le successive traduzioni. Il traduttore invece certe revisioni autorevoli non le dimentica, al punto da farne suoi gli insegnamenti forse anche per sempre, o perlomeno fino al confronto con un altro revisore che sulla medesima questione traduttiva ha una visione opposta a quella del primo.

[36] Si palesa qui un'altra lacuna del mito del «post-editing»: l'aspetto formativo. La revisione di traduzioni non è un'attività che si può apprendere in astratto. Certo, lo studio di libri di testo, manuali e ricerche scientifiche dedicate a questo tema, come pure gli esercizi di revisione nelle scuole di traduzione o le discussioni tra colleghi in occasione di formazioni interne, sono una base fondamentale e imprescindibile per avvicinarsi a quest'attività e approfondirla. Ma l'unico modo in cui un traduttore può imparare concretamente a rivedere le traduzioni altrui, è facendo egli stesso l'esperienza di vedere riviste le proprie traduzioni da altri.

[37] Soltanto confrontando la propria scrittura con la visione di colleghi dotati di un'esperienza più ampia, o comunque diversa dalla propria, il traduttore può innanzitutto progredire come traduttore e in secondo luogo costruirsi un'immagine di che cosa vuol dire «rivedere» una traduzione in tutti i suoi aspetti: intuire come distinguere le particolarità riconducibili alle preferenze 
e idiosincrasie del traduttore, che vanno rispettate, dai passaggi che vanno riformulati poiché errati, infelici o ambigui, e distinguere poi questi, in cui il revisore non può esimersi dal correggere senza venir meno al proprio compito, da quelli invece in cui una riformulazione può essere proposta anche se non indispensabile. Senza dimenticare che molte volte è il revisore a sbagliare o a trarre insegnamento da quanto scritto da un traduttore più competente e creativo di lui.

[38] Revisori non si nasce, lo si diventa per interazione con la scrittura degli altri, e per assimilazione dei riscontri che gli altri ci danno sulla nostra scrittura, e questo processo non è mai compiuto ma, per la natura dialettica della traduzione, è in continuo divenire lungo tutto l'arco della vita professionale di un traduttore-revisore. Ora, in un mondo composto di soli post-editor di traduzioni automatiche, come si pensa di tramandare alle nuove generazioni le conoscenze necessarie per svolgere questa attività con cognizione di causa? Forse invitando le nuove leve a consultare su Youtube qualche tutorial sulla revisione animato da felici pupazzi?

\section{8. È traduzione?}

[39] Tutto sommato è curioso che i risultati ottenuti dai software di traduzione automatica suscitino così tanto scalpore: queste «traduzioni» sono il risultato di un calcolo, e se c'è una cosa che un computer è in grado di fare, e di fare meglio degli esseri umani, sono i calcoli. Peccato solo che la lingua invece non sia il risultato di un calcolo. Come ha detto Francesco Sabatini ${ }^{37}$, essa ci confronta sempre con "problemi spinosi ma vivi», e altrettanto spinosi e vivi sono i problemi con cui ci confronta la traduzione in quanto operazione fatta per la lingua, nella lingua e con la lingua.

[40] La macchina è senza dubbio, ogni traduttore contemporaneo lo sa, un ausilio preziosissimo in questo ambito, proprio per la sua sistematicità, che permette di passare al setaccio grandi quantità di testi di riferimento in tempi brevissimi, di paragonare in modo sistematico testi diversi, di consultare risorse altrimenti difficili da trovare, di sistematizzare la terminologia e via dicendo. Ma la traduzione automatica non è un semplice strumento digitale alla stregua degli altri di cui i traduttori si avvalgono già da vari decenni: nel sostituire interamente la propria logica binaria al complesso processo di costituzione di senso della traduzione, trasforma quest'ultimo in un mero procedimento di commutazione di segni, un calcolo appunto, privo di vita poiché privo di contenuto: «eine kommunikativ operierende Kunst (...), die in dem, was sich konstituiert, sog. den Sinn festhält, der so tot ist wie die Blumen in den gläsernen Gärten Rilkes» ${ }^{38}$.

[41] Per questa sua lacuna di fondo, e non per il fatto che una singola traduzione concreta eseguita dal software contiene o no un errore, la traduzione automatica non potrà mai fornire traduzioni di qualità né, d'altronde, fregiarsi della designazione di «traduzione».

\section{Plurilinguismo istituzionale: quo vadis?}

[42] Ma c'è di più. Il plurilinguismo istituzionale svizzero si fonda sull'equivalenza delle lingue ufficiali. Così recita l'articolo 70 capoverso 1 della Costituzione federale. Questa disposizio-

37 In occasione dell'incontro «Traduzione e italiano» tenuto a Berna il 21 maggio 2015.

38 Altrichter 2016, pag. 83 (corsivo nell'originale). 
ne non dice che le autorità federali si esprimono in una lingua privilegiata le cui formulazioni debbano poi essere tradotte nelle altre lingue ufficiali. Stabilisce invece che lo Stato federale è trilingue nell'espressione della propria volontà. Che cosa significa? Significa, tra l'altro, che nessuna versione linguistica è originale, oppure che nessuna è più originale delle altre, oppure semplicemente che ci sono tre originali. È dire che prima della loro pubblicazione simultanea i tre testi devono contemperarsi reciprocamente affinché sia garantita veramente la pari autorità di ogni versione linguistica. E questo è effettivamente ciò che avviene nel processo traduttivo nelle istituzioni federali svizzere, durante il quale, proprio grazie alla provvisorietà dei testi, ogni versione linguistica può influire sulla forma delle altre ${ }^{39}$. Questo particolare processo traduttivo, che garantisce appunto l'originalità delle tre versioni, si avvale, in particolare nella procedura legislativa, di altri presidî atti ad assicurare l'adempimento dei suoi obiettivi ma, e questo va qui sottolineato con forza, esso eccede totalmente l'attività traduttiva classica, già solo per il fatto che muove da un testo di base che non è immutabile, non è la misura del testo tradotto. La traduzione, in un tale contesto, è sì traduzione, ma è anche molto più, è confronto interlinguistico per trovare nella dialettica trilingue la migliore formulazione di un contenuto che non può pretendere di trovare la sua espressione esatta in una sola lingua ${ }^{40}$. Questa euristicità della traduzione si manifesta chiaramente nella coredazione, ma in realtà innerva e anima tutto il lavoro traduttivo su testi in elaborazione permanente fino alla pubblicazione.

[43] È dire che la traduzione assume in tale contesto la valenza di momento chiave per giungere, attraverso l'analisi dialettica e interlinguistica, a una comunicazione trilingue che sola è capace di ufficialità. Soltanto se intesa come momento essenziale da esperire in tutto il suo spessore - in tutta la sua durata e la sua «fatica» - si può parlare della traduzione istituzionale come di un valore aggiunto per tutte le versioni linguistiche. Momento, appunto, non ostacolo tecnico o pragmatico che occorrerebbe liquidare il più rapidamente possibile.

[44] Quale può essere la pertinenza della traduzione automatica in un siffatto contesto? Essa azzera il processo dialettico di confronto interlinguistico e di critica permutando una serie di grafemi con un'altra serie «equivalente». Tradurre, però, «non è semplicemente una commutazione di codice, ma è una trasposizione di un intero, cioè di un concetto e tutto l'universo mentale a cui pertiene» ${ }^{41}$. In questo senso la traduzione automatica misconosce totalmente le particolarità e il valore aggiunto propri all'esercizio stesso di tale attività, non ha alcuna pertinenza con tale processo e presenta anzi il grande pericolo di insinuare nella gestione del plurilinguismo svizzero una concezione liquidatoria dello stesso, non conforme al dettato costituzionale.

[45] La posizione dell'italiano nella storia della Confederazione dal 1848 a oggi mostra come da sempre vi sia stata una tensione tra gli obiettivi di promozione linguistica da un lato e l'esigenza di un impiego parsimonioso ed efficiente delle risorse pubbliche come pure di una velocizzazione delle procedure dall'altro. La novità sta nel fatto che in Svizzera questo dibattito in passato è sempre stato svolto nella piena consapevolezza della dimensione non solo finanziaria, ma anche politica e sociale che sta alla base di una realtà plurilingue come la nostra, "come si conveniva all'importanza della cosa ${ }^{42}$, mentre oggi un elemento tecnologico come la traduzione automa-

39 Tale processo circolare è descritto segnatamente in EGGER 2019, pagg. 45-48.

40 Su questo particolare approccio traduttivo cfr. EGGER 2019, pag. 37.

41 EGgER 2020

42 Così si esprimeva il Consiglio federale nella sua risposta del 20 novembre 1917 alla richiesta dei «fedeli e cari confederati» (il Consiglio di Stato ticinese) di pubblicare il Foglio federale anche in italiano; Pini 2017, pag. 171. Per 
tica, suscettibile di modificare radicalmente il tessuto istituzionale plurilingue del nostro Paese, sta per così dire accedendo dalla porta di servizio dei bandi di appalto informatici sotto le vesti di un fattorino venuto candidamente a portare quelli che spaccia essere i numeri vincenti della prossima estrazione del lotto.

[46] Ma uno Stato plurilingue può davvero credere di adempiere i suoi obblighi costituzionali mettendo a disposizione delle comunità linguistiche di cui deve farsi garante una lingua generata da macchine, ossia un insieme di grafemi orfani di senso, ossia in definitiva una lingua muta?

Ettore Mjölsnes, Cancelleria federale, Servizi linguistici centrali, Divisione italiana, Berna, e-mail: thor-ettore.mjoelsnes@bk.admin.ch.

L'autore ringrazia Jean-Luc Egger, in particolare anche per l'apporto al capitolo sul plurilinguismo istituzionale.

\section{Bibliografia}

Altrichter, Rudolf (2016): Spiegelungen, Oberhausen.

Altrichter, Rudolf (2020): Metaphysisch Denken, Küssnacht.

Balzaretti, Ugo (2018): Leben und Macht, Weilerswist.

Balzaretti, Ugo (2021): «Georges Canguilhem, «Le cerveau et la pensée»: «intelligenza artificiale» tra senso e volontà», in: Filosofia e attualità, www.philosophie.ch, 12 gennaio 2021.

Buffoni, Franco (2016): Con il testo a fronte. Indagine sul tradurre e l'essere tradotti, Novara.

Decorazioni Pittoriche (1998) = Soldati, Bruno, «Presentazione», in: AAVV., Decorazioni pittoriche in Valmaggia, Cevio e Bellinzona 1998, pagg. 7-8.

De Luca, Erri (2021): A grandezza naturale, Milano.

Egger, Jean-Luc (2019): A norma di (chi) legge. Peculiarità dell'italiano federale, Milano.

EGger, JeAN-Luc (2020): «Intorno al «Normkonzept»: la traduzione italiana del «Gesetzgebungsleitfaden», in: LeGes 31 (2020) 1 .

Egger Jean-Luc/Grandi, Filippo (2013): «Italiano giuridico federale. Un dispaccio dal fronte», in: Jean-Luc Egger/Angela Ferrari/Letizia Lala (a. c. di), Le forme linguistiche dell'ufficialità. L'italiano giuridico e amministrativo della Confederazione Svizzera, Bellinzona 2013, pagg. 213-242.

Nussbaumer, Markus (2020): «Maschinelle Übersetzung», in: LeGes 31 (2020) 3.

Picard, Max (2007): Il mondo del silenzio, Sotto il Monte.

Pini, Verio (2017): Anche in italiano! 100 anni di lingua italiana nella cultura politica svizzera, Bellinzona 2017.

Snozzi, Alfredo (2005): «L'italiano nella legislazione svizzera», in: Marco Borghi (a c. di), Lingua e diritto. La presenza della lingua italiana nel diritto svizzero, Basilea/Ginevra/Monaco, pagg. 317-329.

Sbarbaro, Camillo (1985): «Ars (iners) poetica», in: Id., L'opera in versi e in prosa, Milano, pagg. 421-422.

farsi un quadro di questa consapevolezza nel corso della storia recente del nostro Paese si vedano anche gli altri documenti d'archivio riportati in PINI 2017, pagg. 161 segg. 